\title{
Atypical posterior scleritis mimicking choroidal melanoma
}

Heba M. Alsharif, MBBS, Saad A. Al-Dahmash, MD.

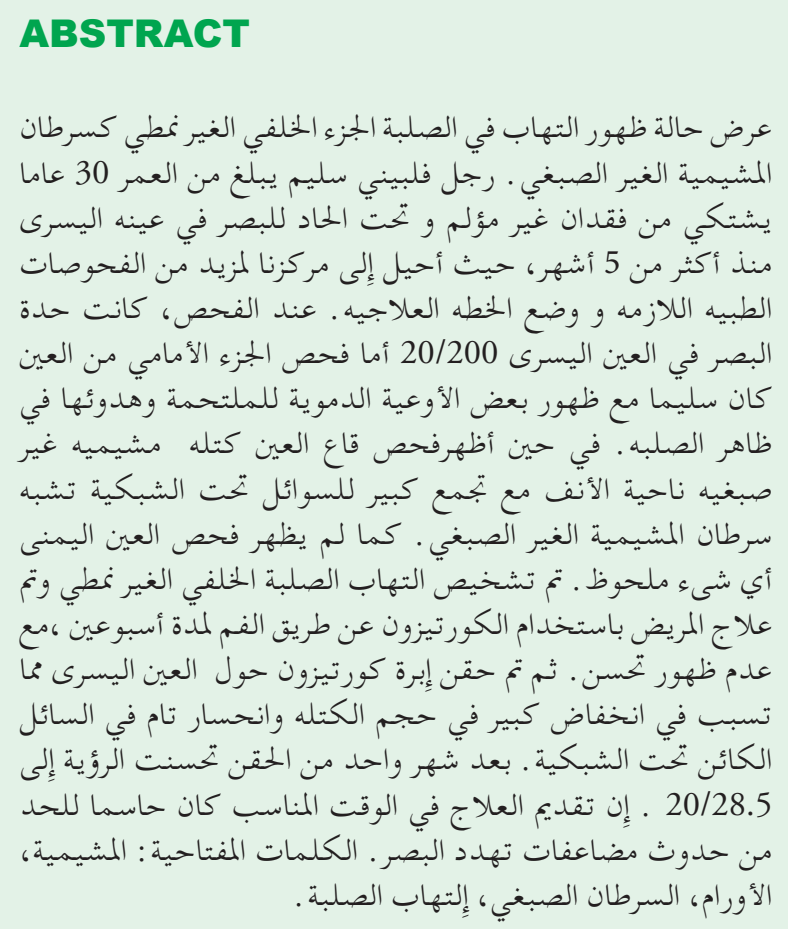

We report a case of atypical posterior scleritis mimicking amelanotic choroidal melanoma. A 30-year-old healthy Filipino man, with a history of painless subacute loss of vision in his left eye over 5 months, was referred to our institute for further workup and management. On examination, visual acuity of the left eye was 20/200. Anterior segment examination yielded unremarkable results, with injected conjunctiva and quiet episcleral blood vessels, while fundus examination revealed non-pigmented nasal choroidal mass, with significant subretinal fluid resembling amelanotic choroidal melanoma. Right eye examination yielded unremarkable results. The patient was diagnosed with atypical posterior scleritis, and treated with oral steroids for 2 weeks, with no improvement. A periocular steroid was then injected to the left eye, causing dramatic reduction in choroidal mass size, and complete resolution of subretinal fluid. The visual acuity improved to $20 / 28.5$ one month after the injection. Timely treatment was crucial for minimizing vision-threatening complications.
Saudi Med J 2018; Vol. 39 (5): 514-518

doi: 10.15537/smj.2018.5.22130

From the College of Medicine, King Saud University, Riyadh, Kingdom of Saudi Arabia.

Received: 21st January 2018. Accepted 23rd April 2018.

Address correspondence and reprint request to: Dr. Saad A. Al-Dahmash, Department of Ophthalmology, Collage of Medicine, King Saud University, Riyadh, Kingdom of Saudi Arabia. E-mail: saldahmash@ksu.edu.sa ORCID: https://orcid.org/0000-0002-9326-0172

$\mathrm{P}$ osterior scleritis, an inflammatory condition affecting the posterior outermost coat of the eye, is relatively uncommon, affecting women more than men. ${ }^{1-10}$ It usually occurs as an isolated ocular disease, yet $19.4 \%$ to $37.7 \%$ cases may be associated with systemic diseases, such as Wegener granulomatosis, systemic lupus erythematosus, and rheumatoid arthritis. ${ }^{4,5}$ Posterior scleritis manifests with a wide array of clinical presentations; thus, it may mimic multiple benign and malignant conditions affecting the posterior ocular structures. ${ }^{1-10}$ Patients usually present subacutely, complaining of one or more of the following symptoms: binocular diplopia in different gazes, periocular pain, decreased vision, lid swelling, and eye protrusion. Clinical examination may reveal restricted motility of the extraocular muscles, and tenderness, proptosis, and/or intraocular inflammation. Posterior segment examination usually shows significant serous (exudative) retinal detachment and/or choroidal fold. Other less common features include: macular edema, subretinal fluid, subretinal mass, and disc edema., ${ }^{4,5}$ The diagnosis of posterior scleritis is based on clinical features, in combination with the findings of essential diagnostic tests, principally the Brightness scan, which usually demonstrates scleral wall thickening and fluid collection in the posterior subtenon space. ${ }^{7}$ Nevertheless, posterior scleritis may impose diagnostic challenges, despite the availability of such conventional ancillary tests, due to its variable clinical manifestations. ${ }^{1,4}$ Various effective therapeutic modalities currently exist 
for the management of posterior scleritis, including non-steroidal anti-inflammatory drugs (NSAIDs), corticosteroids, and immunosuppressive drugs. Nonetheless, failure to establish the correct diagnosis in the first place can misdirect and delay treatment. ${ }^{4,9}$

In this report, we describe an atypical presentation of posterior scleritis masquerading as amelanotic choroidal melanoma that was managed successfully with a single periocular steroid injection.

Case Report. Patient information. A 30-year-old healthy Filipino man, with a history of gradual painless loss of vision in his left eye over 5 months, was referred to our institute as a case of amelanotic choroidal melanoma. His past systemic and ocular history were unremarkable.

Clinical findings. On examination, the visual acuity was 20/20 in the right eye and 20/200 in the left eye. The intraocular pressure was within normal limits for both eyes. On inspection, there was no proptosis in both eyes. Extra-ocular motility was full, with no pain or limitation in all fields of gaze. Pupil examination revealed an afferent pupillary defect in the left eye. Slit-lamp examination of the anterior segment showed injected conjunctiva, quiet anterior episcleral and scleral blood vessels in the left eye, with unremarkable results in the right eye. Right eye fundus examination yielded unremarkable results, whereas the left eye showed a large choroidal non-pigmented lesion nasal to the optic disc, measuring $>10 \mathrm{~mm}$ in basal diameter; optic nerve head swelling; and hyperemia, with extensive inferior exudative retinal detachment (Figures 1A \& 2).

Diagnostic assessment. Diagnostic ultrasonography (B-scan) revealed a choroidal lesion measuring $13 \mathrm{~mm}$ in its largest basal diameter and $3.6 \mathrm{~mm}$ in elevation, with dense echogenicity, no choroidal excavation, and thickened adjacent sclera (Figures 1B \& 2).

The initial workup included: detailed uveitis workup, which revealed negative results for purified protein derivative intradermal skin test; autoimmune serologic screening (rheumatoid factor, antinuclear antibodies, and antineutrophil cytoplasmic antibodies); hepatitis serology; and human immunodeficiency virus and syphilis tests. Radiological tests were performed,

Disclosure. Authors have no conflict of interests, and the work was not supported or funded by any drug company.

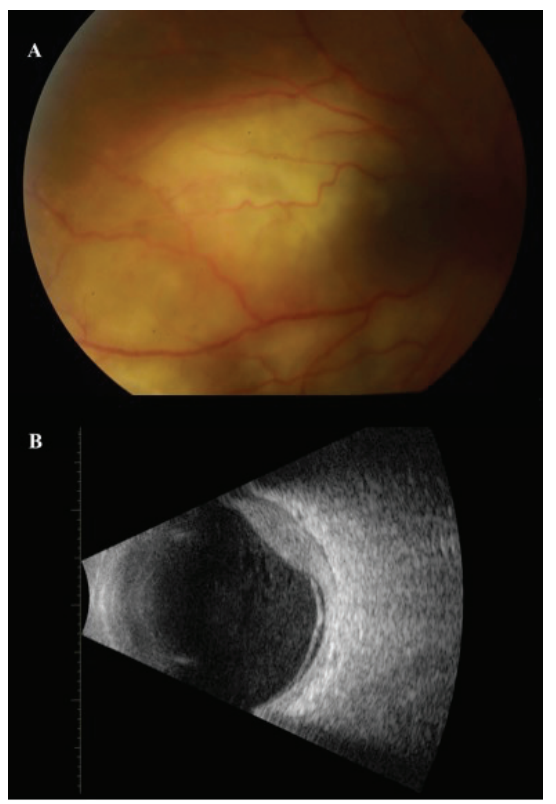

Figure 1 - A) Color photograph of the fundus of the left eye, showing a large choroidal non-pigmented lesion nasal to the optic disc. (B) Brightness scan of the left eye, showing a choroidal lesion with dense echogenicity, thickening of the adjacent sclera, and exudative retinal detachment.

including chest $\mathrm{x}$-ray, chest computed tomography scan, and magnetic resonance imaging of the brain, which yielded unremarkable results.

The absences of hilar lymphadenopathy and focal nodular or infiltrative lung parenchymal densities in chest computed tomography, and normal serum angiotensin converting enzyme level ruled out the possibility of ocular sarcoidosis. Left orbital magnetic resonance imaging showed a mildly enhanced homogenous intraocular retinal mass of $3.2 \mathrm{~mm}$ thickness over the left optic disc and medial to it, associated with effusion superiorly and inferiorly lateral to the optic disc. However, optic disc and extraocular muscles were normal in left orbital magnetic resonance imaging excluding choroidal lymphoma. Moreover, clinical history, examination and diagnostic imaging confirmed the exclusion of primary central nervous system lymphoma that presents with ocular vitritis or retinal pigment epithelium infiltrate and secondary intraocular lymphoma that presents as a part of systemic non-Hodgkin's B cell lymphoma with systemic manifestations, choroidal infiltration, which are both not presented in this healthy gentleman (Figure 2).

Therapeutic intervention. At this stage, the aforementioned findings collectively directed the diagnosis toward atypical idiopathic non-necrotizing posterior scleritis. The patient was orally treated with 


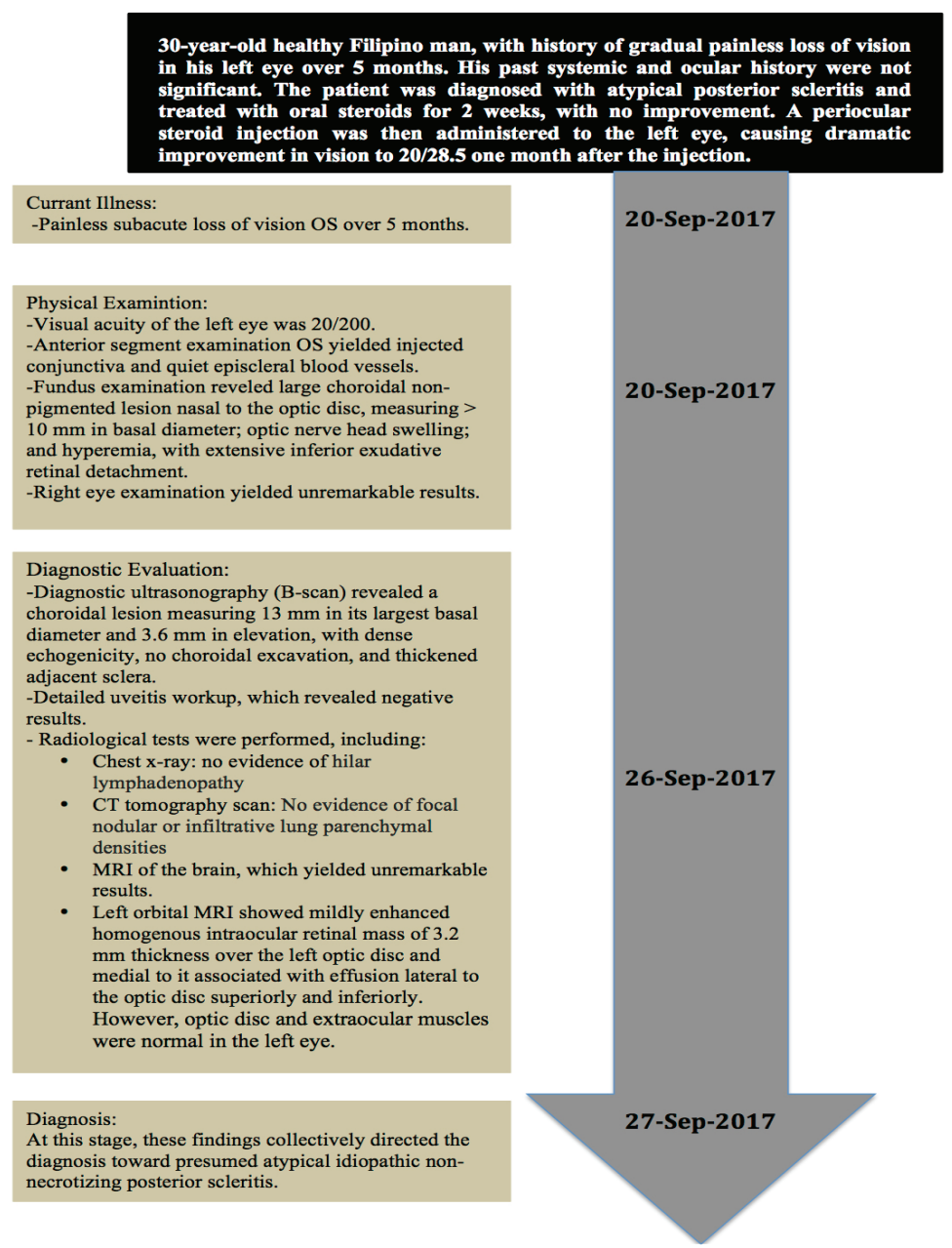

Figure 2 - Timeline summarizing the patient's current illness, physical examination results, diagnostic evaluations, and the final diagnosis.

prednisolone at a dose of $1 \mathrm{mg} / \mathrm{kg}$, for 2 weeks, without any significant improvement; he developed severe gastrointestinal symptoms due to the oral steroids. Therefore, the patient was subsequently administered empirical transseptal periocular triamcinolone acetonide $(40 \mathrm{mg} / \mathrm{ml})$ injection, using a $27 \mathrm{G}$ needle ( 1 inch length), in the inferior temporal quadrant of the orbit away from the globe to avoid injuring the posterior sclera (Figure 3).

Follow-up and outcomes. One month after receiving the periocular steroid injection, the patient showed significant reduction of the choroidal mass, with subretinal gliosis as part of the healing process. The patient exhibited complete resolution of subretinal fluid, and complete recovery of the optic nerve head swelling and hyperemia, and the visual acuity improved to 20/28.5 (Figures 3, 4A \& 4B).
Discussion. The current report presents a case of atypical posterior scleritis mimicking intraocular amelanotic choroidal melanoma. Posterior scleritis may be challenging to diagnose in certain cases as it may present clinically with a subretinal mass due to the localization of the underlying scleral inflammation to a part of the sclera; hence, a wide list of differential diagnoses should be examined thoroughly before reaching a conclusion and proceeding with the treatment. ${ }^{2-7}$ Posterior scleritis most commonly presents with periocular pain, which is reported to occur in 64\% of the cases, unlike our patient, who presented without pain. ${ }^{5}$ Few studies in the literature have reported a presentation similar to that of our patient, where posterior scleritis manifested clinically as a subretinal mass, mimicking the appearance of intraocular tumors, such as amelanotic choroidal melanoma; however, 


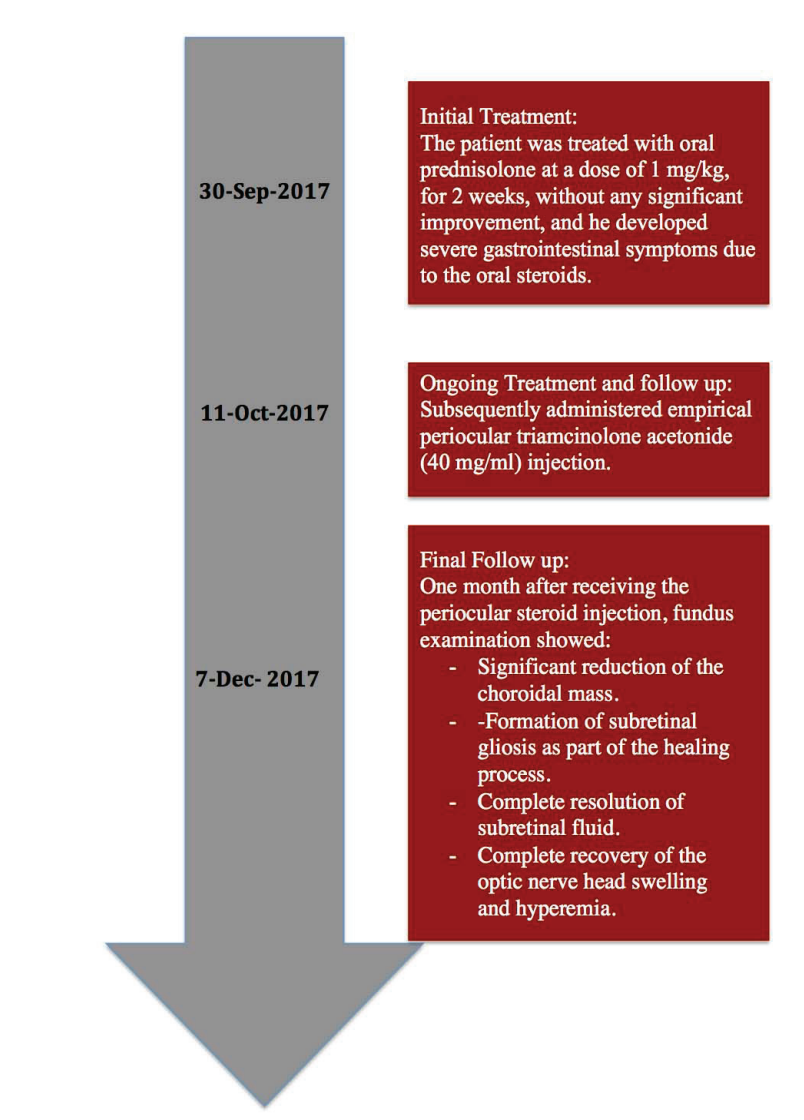

The visual acuity improved to $20 / 28.5$

Figure 3 - Timeline summarizing the sequence of interventions.

ultrasonographic evaluation helped limit the differential diagnosis. ${ }^{3,6,9,10}$ Such studies highlight the importance of B-scan or Amplitude scan as crucial diagnostic tests. ${ }^{4,5}$ Delayed diagnosis may mislead treatment, and expose the patient to vision-threatening and possibly life-threating complications if the underlying systemic illnesses are not diagnosed early in the course of the disease. $^{4}$

The underlying etiology, whether it is autoimmune, infectious, or neoplastic, commonly guides the treatment of posterior scleritis. Treatment options vary depending on the degree of inflammation, ranging from low potency NSAIDs to more potent steroidal drugs, either systemic or locally injectable forms, or other strong immunosuppressive agents. ${ }^{7}$ In general, systemic corticosteroids are considered the first-line treatment for posterior scleritis. ${ }^{1,4}$ On the other hand, locally administered corticosteroids are an effective alternative,

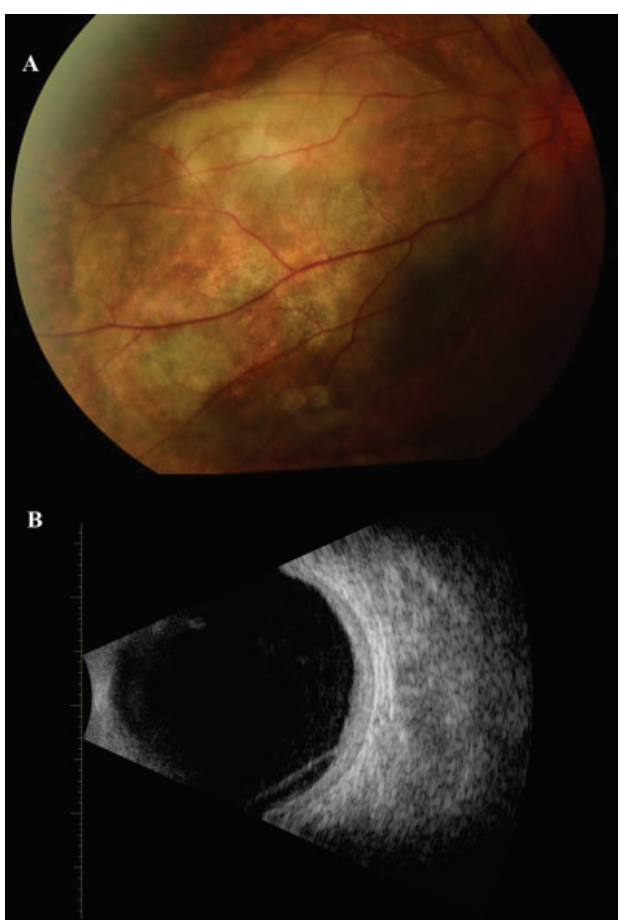

Figure 4 - A) Color photograph of the fundus after treatment, showing significant reduction of the choroidal mass with subretinal gliosis and resolution of subretinal fluid. B) Brightness scan, showing significant reduction in retinochoroidal thickening and exudative retinal detachment.

with fewer undesirable systemic side-effects. ${ }^{5,7}$ However, the administration of subconjunctival steroid injection is contraindicated for necrotizing type of scleritis, as it can lead to scleral melting and subsequent perforation. ${ }^{1}$ As a matter of fact, previous studies have shown that periocular steroid injections achieved good results, and managed to improve vision, and resolve inflammation in patients with posterior scleritis. ${ }^{4}$ The symptoms of the patient in the current study improved dramatically after receiving a single periocular steroid injection.

In conclusion, this case illustrates the importance of early detection and management of posterior scleritis for preserving vision. Although posterior scleritis has variable clinical presentations, considering its atypical presentation as a differential diagnosis for choroidal tumors aids in the diagnosis, and directs toward the appropriate treatment in a timely manner.

Acknowledgment. We would like to thank Editage (www. editage.com) for English language editing.

\section{References}

1. Benson WE. Posterior scleritis. Surv Ophthalmol 1988; 32: 297-316. 
2. Demirci H, Shields CL, Honavar SG, Shields JA, Bardenstein DS. Long-term Follow-up of Giant Nodular Posterior Scleritis Simulating Choroidal Melanoma. Trans $R$ Soc Trop Med Hyg 1973; 67: 276

3. Finger PT, Perry HD, Packer S, Erdey RA, Weisman GD, Sibony PA. Posterior scleritis as an intraocular tumour. British Journal of Ophthalmology 1990; 74: 121-122.

4. McCluskey, Watson PJ, Lightman PG: Posterior scleritis: clinical features, systemic associations, and outcome in a large series of patients. Ophthalmology 1999; 106: 2380-1386.

5. Lavric A, Gonzalez-Lopez JJ, Majumder PD, et al. Posterior Scleritis: Analysis of Epidemiology, Clinical Factors, and Risk of Recurrence in a Cohort of 114 Patients. Ocular immunology and Inflammation 2016; 24: 6-15.
6. Wang J, Lai P, Yang C. Subretinal Mass as a Presenting Sign of Posterior Scleritis: A Case Report. The Kaohsiung Journal of Medical Sciences 2003; 19: 522-524.

7. Sielert L, Harris A, Pyun J, Campbell B, Swan R. Posterior scleritis. Expert Review of Ophthalmology 2016; 11: 475-484.

8. Liu A, Luk F, Chan C. A case of giant nodular posterior scleritis mimicking choroidal malignancy. Indian Journal of Ophthalmology 2015; 63: 919.

9. A Ramanathan, A Gaur. An Atypical Presentation of Posterior Scleritis. The Internet Journal of Ophthalmology and Visual Science 2010; 8: 1-5.

10. Shinisha D P, Suma Elangovan, Sanjeev Kumar Puri, Balaji Ramanathan. Unusual presentation of posterior scleritis. International Journal of Medical Research \& Health Sciences 2016; 5: 54-57. 